\title{
Monetary Policy and the Expected Adjustment Path of Key Variables
}

\author{
James Bullard, President and CEO
}

I t has become commonplace in monetary policy discussions in the United States to say that the Fed is "missing on both sides of its dual mandate." 1 This is often taken to imply that current Fed policy is necessarily far away from an ideal or optimal policy. I do not think that such an inference necessarily follows. The notion that one can easily infer something about the sub-optimality of policy by observing current levels of inflation and unemployment is imprecise. In fact, observing that the Fed is "missing on both sides of the mandate" says little or nothing about the appropriateness of current policy.

\section{It is reasonable to believe that output, employment, and inflation will return to their long-run or targeted values slowly and steadily.}

The Fed famously has a directive that calls for it to maintain stable prices as well as maximum employment, along with moderate long-term interest rates. Since unemployment in the United States is currently high by historical standards, at 7.8 percent in September, many observers argue that the Fed must not be "maximizing employment." In addition, as of August, the personal consumption expenditures price index has increased by about 1.5 percent in the past year. Since the Fed's stated inflation target is 2 percent, then by the numbers, the Fed is "missing on both sides of the mandate." Many then argue that this observation necessarily means that current monetary policy is suboptimal or, worse, badly off track. The argument is that under a proper, optimal monetary policy, when unemployment is above the natural rate, inflation should be above the policymaker's inflation target, not below.

I disagree with this view. I do not think the macroeconomic adjustment literature supports it. Here is my story: The U.S. economy was hit by a large shock in 2008 and
2009. This large shock lowered output and employment far below historical trend levels while also reducing inflation substantially below its target of 2 percent. The key question that must be addressed is this: How do we expect these variables to return to their long-run or targeted values under appropriate monetary policy?

The answer from the macroeconomic literature is that it is reasonable to believe that output, employment, and inflation will return to their long-run or targeted values slowly and steadily. We refer to this type of convergence process as being monotonic: The shock knocks key variables off their long-run values, and the variables gradually return after the shock, provided the policymaker runs a reasonable monetary policy. Other dynamics would be disconcerting. We would not want or expect key variables to gyrate wildly about their long-run values.

Given this type of adjustment, then, it is clear that the Fed will be "missing on both sides of its mandate" during the entire time it takes the economy to return to normal, even when the monetary policy in place is very good. In fact, missing on both sides of the mandate is exactly what one would expect under an appropriate monetary policy. Moreover, the literature suggests that the adjustment times are quite long.

Let's consider the medium-sized macroeconomic framework of Smets and Wouters (2007). This is an important benchmark model; and, while we could argue about the details, I think it will serve to make my point. In the Smets and Wouters dynamic stochastic general equilibrium (DSGE) model there are many shocks, and there is a monetary policymaker that follows a Taylor-type monetary policy rule not unlike ones used in actual policy discussions. The authors estimate their model using postwar U.S. data, and they also report results for subsamples including the post-1984 data. Importantly, what the authors are estimating is a general equilibrium for the economy, which includes monetary policy. 


\section{Estimated Mean Impulse Responses to "Demand" Shocks}
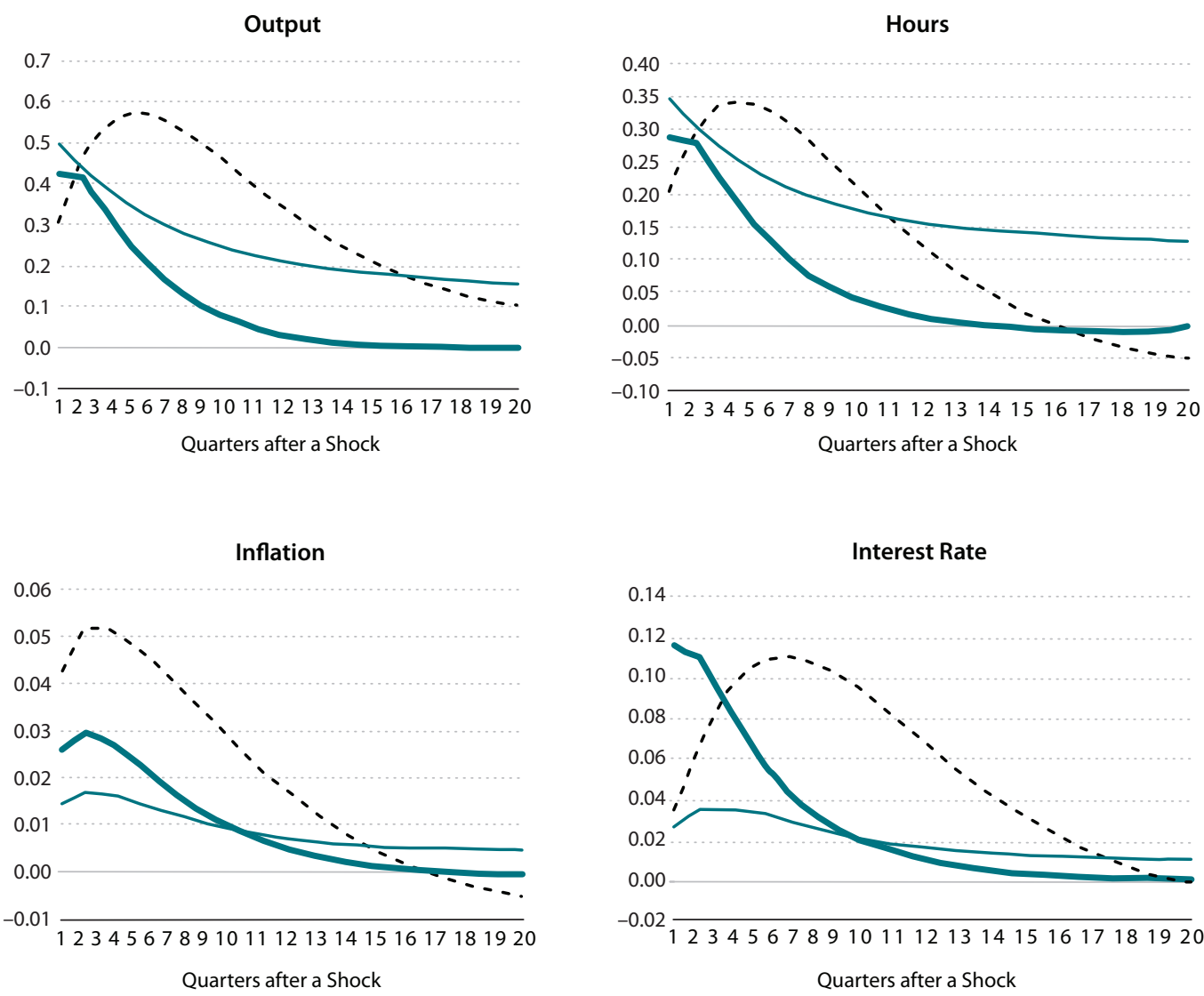

NOTE: The bold solid line shows the response to a risk premium shock, the thin solid line shows the response to an exogenous spending shock, and the dashed line shows the response to an investment shock.

SOURCE: Reproduced with permission, from Smets and Wouters (2007).

How does the economy adjust in the Smets and Wouters model? The chart is Figure 2 from their paper. The authors plot the reaction of key macroeconomic variables to three types of shocks in their model that might be thought of as demand shocks. Variables are reported as deviations from a steady-state value, so that zero represents a return to normal. The variables include inflation and a labor market variable-hours worked. Time is measured in quarters. The shock is a positive one-output and hours go up in response-but the story is merely transposed for a negative shock (i.e., flip the figures upside down).

The reaction of all variables is essentially monotonic beyond the hump in these graphs, at least through year four. (That is, the adjustment does not show much of a tendency to oscillate about the long-run value.) For all three types of demand shocks, the Fed would be "missing on both sides of the dual mandate" almost all of the time as the economy recovers from the shock. If the shock were negative, hours would be too low (unemployment too high), and inflation would be too low every quarter for many years. Yet the monetary policy embedded in this general equilibrium is a Taylor-type policy rule that has often been argued to closely approximate the optimal monetary policy in frameworks such as this one. ${ }^{2}$ It is in this sense that I do not think merely observing where inflation and unemployment are relative to targets or long-run levels at a point in time is telling us very much about whether the monetary policy in use is the appropriate one or not.

Other models may suggest different dynamics, of course, and I am not saying that the Smets-Wouters framework is ideal. In actual economies, other shocks occur during the adjustment process, muddying the waters. 
Furthermore, current U.S. monetary policy has unconventional features that have not been present in the past. But still, many estimated models do suggest that key macroeconomic variables adjust to shocks in a one-sided way under good policy. When there is overshooting, as occurs in year five for the investment shock in Smets and Wouters' Figure 2, both the labor market and inflation variables overshoot at the same time-one would still be "missing on both sides of the mandate" in this case but in the opposite direction. All in all, I do not think it makes much sense to simply cite the values of variables along an adjustment path as evidence of inappropriate policy. To make a proper assessment one has to consider the full implications of a model.

\section{Notes}

${ }^{1}$ For related reading, see Bullard's Financial Times op-ed on September 19, 2012 ("Patience Needed for Fed's Dual Mandate"), and column forthcoming in the October 2012 issue of The Regional Economist ("The Fed Is Not 'Missing on the Dual Mandate"').

2 One can investigate optimal-control monetary policy assuming credible commitment in this model, taking the non-policy parameters as estimated by Smets and Wouters. This type of monetary policy changes these impulse response functions but still leaves goal variables "missing on both sides of the mandate" in many situations. I thank Robert Tetlow for investigating this issue in response to an earlier draft.

\section{Reference}

Smets, Frank and Wouters, Rafael. "Shocks and Frictions in U.S. Business Cycles: A Bayesian DSGE Approach." American Economic Review, 2007, 97(3), pp.586-606. 